\title{
IMPACT AND QUASISTATIC LIMIT IN THE QUANTUM-PATH THEORY OF PRESSURE BROADENING
}

\author{
J. Butaux \\ Laboratoire de Spectroscopie Atomique, Département de Recherches Physiques (L.A. 71), Université P. et \\ M. Curie, 4, place Jussieu, Tour 22, 75230 Paris Cedex 05, France \\ G. NIENHUIS \\ Fysisch Laboratorium, Rijksuniversiteit te Utrecht, Utrecht, The Netherlands \\ and \\ F. SCHULLER \\ Laboratoire des Interactions Moléculaires et des Hautes Pressions, CNRS Université Paris-Nord, Av. J.B. \\ Clément, 93430 Villetaneuse, France \\ (Received 16 June, 1978)

\begin{abstract}
'Abstract-The quantum-path theory of collision broadening involves a correlation function which can be calculated by infinite-order perturbation theory. From the expression obtained, both the impact and the quasistatic limit are easily derived. Application of the WKB method is discussed for the impact result.
\end{abstract}

\section{INTRODUCTION}

IN MOST theories of pressure broadening of spectral lines, one makes the assumption that the motion of perturbers with respect to the absorbing (or emitting) atom can be treated classically (classical path theories). The interaction Hamiltonian then becomes a given function of time, depending on the trajectory followed by the atoms during a collision. Very often the problem is further simplified in that straight line trajectories are used. Only a few authors ${ }^{(1)}$ have tried to refine the model by introducing curved trajectories corresponding to Newtonian motion governed by the interatomic potential. However, this procedure leads to ambiguous results since the interatomic potential changes during the optical transition and it is not clear whether the ground or the excited state potential or even some intermediate potential are to be used. Moreover, except in special cases, this type of theory violates Kirchhoff's law, as has been shown by NienhuIs. ${ }^{(2)}$ These difficulties disappear if the motions of the perturbers are treated quantum-mechanically (quantum-path theories).

The first rigorous treatment using a quantum-path theory has been given by Baranger, who derived expressions for the broadening and shift of a spectral line in the impact limit. ${ }^{(3)}$ In this paper, we use Baranger's model to derive an expression, based on infinite-order perturbation theory, yielding not only the impact limit but the quasistatic limit as well. Furthermore, in the case of the impact limit, the WKB approximation is discussed with reference to the purely quasi-classical results. In the following derivation, we consider absorption spectra and we further assume for simplicity that all interaction potentials are spherically symmetric.

\section{GENERAL EXPRESSION FOR THE CORRELATION FUNCTION}

Our general formulation is the same as in Baranger's work, ${ }^{(3)}$ with the following approximations:

(i) Born-Oppenheimer factorization of the electronic wave function of the atom and of the translational wave function describing the relative motion of the perturbers. $\dagger$

(ii) Space-fixed atoms and independent perturber motion.

†The word "atom" refers to the atom performing the optical transition under study. 
The absorption-line profile can be expressed by the relation

$$
I(\omega)=(1 / \pi) \operatorname{Re} \int_{0}^{x} \mathrm{~d} \tau \mathrm{e}^{\mathrm{i} \omega \tau} \phi(\tau),
$$

where $\phi(\tau)$ is the autocorrelation function of the dipole moment.

In the simple, non-degenerate case with additive perturbations, $\phi(\tau)$ is given by $\phi(\tau)=$ $[\varphi(\tau)]^{N}$, where $N$ is the number of perturbers; the binary correlation function $\varphi(\tau)$ can be written in the form ${ }^{(3)}$

$$
\varphi(\tau)=1-g(\tau) / \Omega
$$

with $\Omega$ being the normalization volume. Taking $\Omega$ to infinity, the correlation function becomes

$$
\phi(\tau)=\exp [-n g(\tau)], \quad n=N / \Omega .
$$

We express the binary correlation function $\varphi(\tau)$ in terms of wave functions associated with the perturber motion for both the ground $(g)$ and excited $(e)$ states of the atom. According to Ref. (3), $\varphi(\tau)$ is given by

$$
\varphi(\tau)=\sum_{\mathbf{k}} \rho_{k} \varphi_{\mathbf{k}}(\tau)=\left\langle\boldsymbol{\varphi}_{\mathbf{k}}(\tau)\right\rangle_{\mathbf{k}}
$$

and

$$
\varphi_{\mathrm{k}}(\tau)=\sum_{\mathbf{k}}\left\langle\chi_{g \mathbf{k}} \mid \chi_{e \mathbf{k}}\right\rangle\left\langle\chi_{e \mathbf{k}} \mid \chi_{g \mathbf{k}}\right\rangle \mathrm{e}^{-i\left(E_{k^{\prime}}-E_{k} \mid \tau / h\right.}
$$

Here $\rho_{k}$ is the statistical weight-factor and $\mathbf{k}, E_{k}$ and $\mathbf{k}^{\prime}, E_{k^{\prime}}$ are the momenta and energies of the stationary translational states $|\chi\rangle$, respectively. For simplicity, we set $\hbar=1$. Summing over intermediate states $\mathbf{k}^{\prime}$, one can write

$$
\varphi_{\mathbf{k}}(\tau)=\mathrm{e}^{i E_{k} \tau}\left\langle\chi_{\boldsymbol{g} \mathbf{k}}\left|\mathrm{e}^{-i H_{e^{\tau}}}\right| \chi_{g \mathbf{k}}\right\rangle ;
$$

here $H_{e}$ is the Hamiltonian associated with the excited state. Let us define the following Green function:

$$
G^{+}(\mathbf{k}, \tau)=(1 / i)\left\langle\chi_{g \mathbf{k}}\left|\mathrm{e}^{-i H_{c} \tau}\right| \chi_{g \mathbf{k}}\right\rangle \theta(\tau)
$$

$\theta(\tau)$ being the Heaviside step function.

According to Eq. (1), it is sufficient to know the correlation function for positive values of $\tau$. Hence, we may consider instead of $\varphi_{\mathbf{k}}(\tau)$ a function defined as

$$
\varphi_{\mathrm{k}}{ }^{+}(\tau)=\varphi_{\mathbf{k}}(\tau) \theta(\tau)=\mathrm{e}^{i E_{k} \tau} i G^{+}(\mathbf{k}, \tau)
$$

From Eq. (4), the Green function in $\omega$-spacc.

is given by

$$
G^{+}(\mathbf{k}, \omega)=\int_{-\infty}^{+\infty} \mathrm{d} \tau \mathrm{e}^{i \omega \tau} G^{+}(\mathbf{k}, \tau)
$$

$$
G^{+}(\mathbf{k}, \omega)=\lim _{\delta \rightarrow 0^{+}}\left\langle\chi_{g \mathbf{k}}\left|\frac{1}{\omega-H_{e}+i \delta}\right| \chi_{g \mathbf{k}}\right\rangle .
$$

From Eqs. (3a) and (5), we can write

$$
\varphi^{+}(\tau)=\sum_{k} \rho_{k} \mathrm{e}^{i E_{k} \tau}(i / 2 \pi) \int_{-\infty}^{+\infty} \mathrm{d} \omega \mathrm{e}^{-i \omega \tau} G^{+}(\mathbf{k}, \omega) .
$$


In the following section, the quantity $G^{+}(\mathbf{k}, \omega)$ will be evaluated by using a perturbation expansion.

\section{EXPANSION OF $G^{+}(\mathbf{k}, \omega)$}

The starting point will be the following identity between operators:

$$
1 / A=1 / B+(1 / B)(B-A)(1 / A),
$$

which can be written as the infinite sum

$$
1 / A=1 / B+(1 / B)(B-A)(1 / B)+(1 / B)(B-A)(1 / B)(B-A)(1 / B)+\cdots
$$

In the present case, $A=\omega-H_{e}+i \delta, B=\omega-H_{g}+i \delta ; B-A=H_{e}-H_{g}=V_{e}-V_{g}$. Here, $H_{e}$ and $H_{8}$ are the Hamiltonians for the excited (e) and the ground $(g)$ states, respectively, and $V_{e}$ and $V_{g}$ are the corresponding potentials. The difference potential $V_{e}-V_{R}$ will be denoted by $V$.

We thus obtain an infinite-order perturbation expansion for $G^{+}(\mathbf{k}, \omega)$, viz.

$$
G^{+}=G_{0}^{+}+G_{1}^{+}+\ldots+G_{n}^{+}+\ldots=\sum_{n=0}^{\infty} G_{n}^{+},
$$

where ${ }^{\dagger}$

$$
G_{n}^{+}=\frac{1}{\left(\omega-E_{k}+i \delta\right)^{2}} \sum_{\mathbf{k}_{1}, \mathbf{k}_{2} \ldots k_{n-1}} \frac{V_{\mathbf{k k}_{1}} V_{\mathbf{k}_{1} \mathbf{k}_{2}} \ldots V_{\mathbf{k}_{n-1} \mathbf{k}}}{\left(\omega-E_{k_{1}}+i \delta\right) \ldots\left(\omega-E_{\mathbf{k}_{n-1}}+i \delta\right)}
$$

In Eq. (9), $V_{\mathbf{k}_{i} \mathbf{k}_{j}}$ is the matrix element $\left\langle\chi_{\varepsilon \mathbf{k}_{i}}|V| \chi_{g \mathbf{k}_{j}}\right\rangle$. Figure 1 shows diagrammatically the expansion for the Green function.

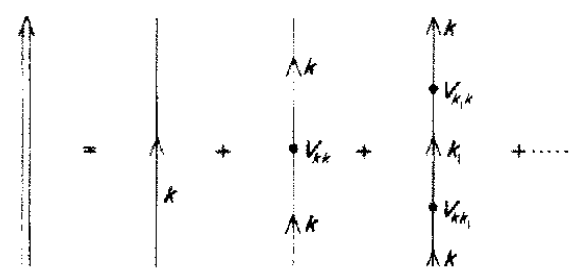

Fig. 1. Diagrammatic expansion of the Green function. The dots represent matrix elements and the lines between them are the zeroth-order Green functions.

Returning to the correlation function, we write, using the definition of Eq. (5) and the inverse relation (6),

$$
\varphi_{\mathrm{k}}(\tau)=1+(i / 2 \pi) \int_{-\infty}^{+\infty} \mathrm{d} \omega\left[\mathrm{e}^{i\left(E_{k}-\omega\right) \tau}-1\right] G^{+}(\mathbf{k}, \omega), \quad \tau>0
$$

which is obtained by introducing the additional relation

$$
(i / 2 \pi) \int_{-\infty}^{+\infty} \mathrm{d} \omega G^{+}(\mathbf{k}, \omega)=\varphi_{\mathbf{k}}(0)=1 .
$$

Equation (10) can be rewritten in terms of the expansion (9), as follows:

$$
\varphi_{\mathbf{k}}(\tau)=1+(i / 2 \pi) \sum_{n=1}^{\infty} \int_{-\infty}^{+\infty} \mathrm{d} \omega\left[\mathrm{e}^{i\left(E_{k}-\omega\right) \tau}-1\right] G_{n}^{+}(\mathbf{k}, \omega), \quad \tau>0
$$

It is seen that the term with $n=0$ gives no contribution.

It is tacitly understood that $\lim \delta \rightarrow 0^{+}$has to be taken. 
In the expression for $G_{n}{ }^{+}(\mathbf{k}, \omega)$, as given by Eq. (9), one of the factors $\omega-E_{k}+i \delta$ in the denominator may be replaced by $\omega-E_{k}$ because the factor $\mathrm{e}^{i\left(E_{k}-\omega\right) \tau}-1$ of Eq. (11) goes to zero as $E_{k}-\omega$ for $\omega \rightarrow E_{k}$ so that no additional divergence appears. We then introduce the following decomposition:

$$
\begin{aligned}
& \frac{1}{\left(\omega-E_{k}+i \delta\right)\left(\omega-E_{k}\right)\left(\omega-E_{k_{1}}+i \delta\right) \ldots\left(\omega-E_{k_{n-1}}+i \delta\right)} \\
& \quad=\frac{1}{\omega-E_{k}+i \delta}\left(\frac{A_{0 n}}{\omega-E_{k}}+\sum_{\nu=1}^{n} \frac{A_{\nu n}}{\omega-E_{k_{v}}+i \delta}\right) .
\end{aligned}
$$

We now perform the integrations over $\omega$, which leads to the following results for $\delta \rightarrow 0^{+}$and $\tau>0$ :

$$
\begin{aligned}
& \int_{-\infty}^{+\infty} \mathrm{d} \omega \frac{\mathrm{e}^{i\left(E_{k}-\omega\right) \tau}-1}{\left(\omega-E_{k}+i \delta\right)\left(\omega-E_{k}\right)}=-2 \pi \tau, \\
& \int_{-\infty}^{+\infty} \mathrm{d} \omega \frac{\mathrm{e}^{i\left(E_{k} \omega\right) \tau}-1}{\left(\omega-E_{k}+i \delta\right)\left(\omega-E_{k_{v}}+i \delta\right)}=\frac{2 \pi i}{E_{k}-E_{k_{k}}}\left[\mathrm{e}^{i\left(E_{k}-E_{k_{k}}\right) / \tau}-1\right] .
\end{aligned}
$$

Using these relations together with Eqs. (9), (11), and (12), we finally obtain

$$
\begin{aligned}
\varphi_{\mathbf{k}}(\tau)=1-i V_{\mathbf{k} \mathbf{k}} \tau+(i / 2 \pi) \sum_{n=2}^{\infty} \sum_{k_{1}} \sum_{\kappa_{n-1}} V_{\mathbf{k} \mathbf{k}_{1}} \ldots V_{\mathbf{k}_{n-1}} \\
\times\left\{-2 \pi \tau A_{\theta n}+2 \pi i \sum_{\nu=1}^{n-1} \frac{A_{\nu n}}{E_{k}-E_{k_{v}}}\left(\mathrm{e}^{i\left(E_{k}-E_{k_{k}}\right) \tau}-1\right)\right\} \text { for } \tau>0
\end{aligned}
$$

From this general equation, both the impact and the quasistatic limit can be obtained, as will now be shown.

\section{THE IMPACT LIMIT}

Up to this stage, the only approximations are (i) and (ii). We now add the impact approximation, according to which only large correlation times have to be considered. This procedure amounts to neglecting, on the right-hand side of Eq. (14), the term that is not proportional to $\tau$ since, at very large $\tau$, the other term will obviously be dominating.

Replacing $A_{0 n}$ by

$$
A_{0 n}=1 /\left(E_{k}-E_{k_{1}}+i \delta\right) \ldots\left(E_{k}-E_{k_{n-1}}+i \delta\right),
$$

we obtain the expression for the binary correlation function in the impact limit, viz.

$$
\varphi_{\mathbf{k}}(\tau)=1-i \tau\left(V_{\mathbf{k} \mathbf{k}}+\sum_{n=1}^{\infty} \sum_{\mathbf{k}_{1}, \ldots k_{n-1}} \frac{V_{\mathbf{k} \mathbf{k}_{1}} V_{\mathbf{k}_{1} \mathbf{k}_{2}} \ldots V_{\mathbf{k}_{n-1} \mathbf{k}}}{\left(E_{k}-E_{k_{1}}+i \delta\right) \ldots\left(E_{k}-E_{k_{n-1}}+i \delta\right)}\right) .
$$

This expression may be identified with the following relation given by BARANGER: ${ }^{(3)}$

$$
\varphi_{\mathrm{k}}(\tau)=1-i \tau\left\langle\chi_{g k}|V| \chi_{e k}\right\rangle
$$

In order to show that the last two expressions are equal, we introduce the following expansion known from scattering theory: $:^{(5)}$

$\left|\chi_{e \mathbf{k}}\right\rangle=\left|\chi_{g \mathrm{k}}\right\rangle+\frac{1}{E_{k}-H_{g}+i \delta} V\left|\chi_{g k}\right\rangle+\frac{1}{E_{k}-H_{g}+i \delta} V \frac{1}{E_{k}-H_{g}+i \delta} V\left|\chi_{g k}\right\rangle+\ldots$

The resulting expansion for $\varphi_{\mathbf{k}}(\tau)$ can be cast in the form of Eq. (15) by inserting intermediate states and acting on these with the operator $H_{g}$. 
By combining Eq. (16) with Eqs. (2a, b), we see that the correlation function for the $N$-perturber system may be written in the form

$$
\phi(\tau)=\exp \left(-i \tau n \Omega\left(\chi_{g \mathrm{k}}|V| \chi_{e \mathrm{k}}\right)\right)
$$

By substituting this into the expression (1), we get a Lorentzian profile. If we then express the quantity $\left\langle\chi_{g \mathrm{k}}|V| \chi_{e \mathrm{k}}\right\rangle$ in terms of phase-shifts, ${ }^{(6)}$ the following results for impact broadening and shift, known as Lindholm's formulae, are obtained:

$$
\begin{aligned}
& \text { shift }=-n \pi(1 / m)\left\langle(1 / k) \sum(2 l+1) \sin \left[2\left(\delta_{e k l}-\delta_{g k l}\right)\right]\right\rangle_{a v}, \\
& \text { width }=n 2 \pi(1 / m)\left\langle(1 / k) \sum(2 l+1)\left(1-\cos \left[2\left(\delta_{e k l}-\delta_{g k l}\right)\right]\right)\right\rangle_{a v} .
\end{aligned}
$$

Here $l$ is an angular momentum quantum number, which appears if the wave functions are expanded into partial waves and the phase-shifts are defined by the asymptotic expressions of the corresponding radial parts, which are proportional to

$$
(1 / k r) \sin \left[k r-(\pi / 2) l+\delta_{e, g k l}\right]
$$

for large interatomic distances $r$.

In order to evaluate the phase-shifts $\delta_{e, g k l}$, one possibility is to use the WKB-method, as will be shown later. However, except in the quasiclassical limit, it is not easy to obtain numerical results by applying the above equations.

\section{THE QUASISTATIC LIMIT}

In Eq. (14) we introduce the expansion

$$
\mathrm{e}^{i\left(E_{k}-E_{k_{\nu}}\right) \tau}-1=\sum_{\mu=1}^{\infty}(1 / \mu !)(i \tau)^{\mu}\left(E_{k}-E_{k_{\nu}}\right)^{\mu}
$$

We then have

$$
\varphi_{\mathbf{k}}(\tau)=1-i \tau V_{\mathbf{k k}}-\sum_{n=2}^{\infty} \sum_{k_{1}, \ldots k_{n-1}} V_{\mathbf{k} \mathbf{k}_{1}} \ldots V_{\mathbf{k}_{n-1} \mathbf{k}}\left(i \tau A_{0 n}+\sum_{\nu=1}^{n-1} \sum_{\mu=1}^{\infty} B_{\nu n}^{\mu-1}(i \tau)^{\mu} / \mu !\right)
$$

with

$$
B_{\nu n}^{\mu-1}=A_{\nu n}\left(E_{k}-E_{k_{v}}\right)^{\mu-1}
$$

Inside the parentheses, the term $i \tau A_{0 n}$ combines with the terms corresponding to $\mu=1$ to give $i \tau \sum_{\nu=0}^{n-1} A_{\nu n}$, which is zero in view of the definition of the $A_{v n}$. On the other hand, the coefficients $B_{v n}^{\mu-1}$, which satisfy the relation (21), can be defined by the following expansion:

$$
-\frac{\left(E_{k}-\omega\right)^{\mu-2}}{\left(\omega-E_{k_{1}}+i \delta\right) \ldots\left(\omega-E_{k_{n-1}}+i \delta\right)}=\sum_{\nu=1}^{n-1} \frac{B_{\nu n}^{\mu-1}}{\omega-E_{k_{\nu}}+i \delta} \quad(2 \leqslant \mu \leqslant n) .
$$

Straightforward consequences of this procedure are listed below:

for

$$
\mu<n, \quad \sum_{\nu=1}^{n-1} B_{\nu}^{\mu-1}=0 ;
$$

for

$$
\mu=n, \quad \sum_{\nu=1}^{n-1} B_{\nu}^{\mu-1}=(-1)^{n-1} .
$$


Changing the order of the summation in Eq. (20) and using Eq. (23), we obtain

$$
\begin{aligned}
\varphi_{\mathbf{k}}(\tau)=1-i \tau V_{\mathbf{k} \mathbf{k}}+\sum_{n=2}^{\infty} \sum_{\mathbf{k}_{1} \ldots \mathbf{k}_{n-1}} V_{\mathbf{k} \mathbf{k}_{1}} \ldots V_{\mathbf{k}_{n-1} \mathbf{k}}(-i \tau)^{n} / n ! \\
\quad-\sum_{n=2}^{\infty} \sum_{\mathbf{k}_{1} \ldots \mathbf{k}_{n-1}} V_{\mathbf{k} \mathbf{k}_{1}} \ldots V_{\mathbf{k}_{n-1} \mathbf{k}} \sum_{\mu=n+1}^{\infty} \sum_{\nu=1}^{n-1} B_{v n}^{\mu-1}(i \tau)^{\mu} / \mu ! .
\end{aligned}
$$

The second and third terms of Eq. (24) can be written as

$$
\sum_{n=1}^{\infty}\left(V^{n}\right)_{\mathbf{k k}}(-i \tau)^{n} / n !
$$

We finally obtain the following expansion:

$$
\varphi_{\mathbf{k}}(\tau)=\left(\mathrm{e}^{-i V_{\tau}}\right)_{\mathbf{k} \mathbf{k}}-\sum_{n=2}^{x} \sum_{\mathbf{k}_{1} \ldots \mathbf{k}_{n-1}} V_{\mathbf{k} \mathbf{k}_{1}} \ldots V_{\mathbf{k}_{n-1}} \sum_{\mu=n+1}^{\infty} \sum_{\nu=1}^{n-1} B_{v n}^{\mu-1}(i \tau)^{\mu} / \mu !
$$

We now take the quasistatic limit by considering $\tau$ as a vanishingly small quantity. If we then compare in Eq. (26) the first and the second terms on the right-hand side, we see that, for a given order $n$ in the potential, the first term yields a factor $\tau^{n}$ whereas the second is of higher order in $\tau$ with factors $\tau^{n+1}, \tau^{n+2}$, etc. . Consequently, in the quasistatic limit, we disregard the second term and write

$$
\varphi_{\mathbf{k}}(\tau)=\left(\mathrm{e}^{-i V_{t}}\right)_{\mathbf{k} \mathbf{k}}
$$

Using Eqs. (2a, b) and Eq. (3a), we thus obtain the following relations:

$$
\begin{aligned}
\varphi(\tau) & =\sum_{k} \rho_{k} \varphi_{\mathbf{k}}(\tau)=\operatorname{Tr}\left(\rho \mathrm{e}^{-i V_{\tau}}\right) \\
& =1-(1 / \Omega) \operatorname{Tr}\left(\Omega \rho\left(1-\mathrm{e}^{-i V_{\tau}}\right)\right), \\
\phi(\tau) & =\exp \left[-n \operatorname{Tr}\left(\Omega \rho\left(1-\mathrm{e}^{i V_{\tau}}\right)\right]\right.
\end{aligned}
$$

In the last equation, the normalization volume drops out if properly normalized wave functions are used to evaluate the trace.

The correlation function given by Eq. (29) is equivalent to the well-known quasiclassical result. ${ }^{(6)}$

\section{THE WKB APPROXIMATION IN THE IMPACT IIMIT}

Explicit calculations of half-width and shift from Lindholm's formulae (19) are not easy to perform. We tried to obtain approximate results by using the WKB method. According to this method, the quantum mechanical phase-shifts are given by the following expressions: ${ }^{(7)}$

$$
\delta_{e k l}-\delta_{g k l}=\int_{r_{e}}^{\infty} F_{e} \mathrm{~d} r-\int_{r_{g}}^{\infty} F_{\mathrm{g}} \mathrm{d} r+\eta_{e}-\eta_{g}
$$

The different quantities entering this equation are defined as follows:

$$
F_{e}=k\left[1-l(l+1) / k^{2} r^{2}-V_{e}(r) / E\right]^{1 / 2}
$$

with a similar expression for $F_{g} ; E$ is the total energy of the relative motion; $r_{e}$ and $r_{g}$ are the classical turning-points for the potentials $V_{e}$ and $V_{g}$, respectively.

Quantum-mechanically, there can be cases with three turning points and then the additional terms $\eta_{e}$ and $\eta_{g}$ of Eq. (30) must be calculated ${ }^{(7)}$. However, we found that these terms had only a small influence on the total cross-section. 
Using the relations given above, we have performed detailed calculations for the systems $\mathrm{Hg}-\mathrm{He}^{3}$ and $\mathrm{Hg}-\mathrm{He}^{4}$ and have found considerable deviations from the purely classical results. In fact, one can show that, in order to obtain the usual classical expression from Eq. (30), considerable simplifications have to be made. Setting $r_{e} \sim r_{g}$ and neglecting $\eta_{e}$ and $\eta_{g}$, we write

$$
\delta_{e k l}-\delta_{g k l}=\int_{r_{g}}^{\infty}\left(F_{e}-F_{g}\right) \mathrm{d} r
$$

Furthermore, we assume $V=V_{e}-V_{g} \ll V_{g}$ and, by using Eq. (31), we obtain to the first order in $V$,

$$
\int_{r_{g}}^{\infty}\left(F_{e}-F_{g}\right) \mathrm{d} r=(k / 2 E) \int_{r_{g}}^{\infty} V \mathrm{~d} r /\left[1-l(l+1) / k^{2} r^{2}-V_{g} / E\right]^{1 / 2}
$$

In the classical limit, where $l(l+1) / k^{2} \rightarrow b^{2}(b=$ impact parameter $), k / 2 E \rightarrow 1 / v_{0}\left(v_{0}=\right.$ initial relative velocity), Eq. (32) reduces to

$$
\delta_{e}-\delta_{g} \rightarrow \eta_{c} / 2=\left(1 / v_{0}\right) \int_{r}^{\infty} V \mathrm{~d} r /\left(1-b^{2} / r^{2}-V_{g} / E\right)^{1 / 2},
$$

which has been used by several authors in their calculations of pressure broadening and shift. ${ }^{(1)}$

Although Eq. (33) can be obtained from the general expression in a formal manner, its application is not justified in most practical cases because of the restrictive approximations that were made. In particular, it is doubtful that the difference potential can be treated as negligibly small compared to $V_{g}$. Therefore, large deviations have to be expected if the correct expression (30) is employed.

\section{REFERENCES}

1. L. Herman and G. COUlaud, JQSRT 10, 571 (1970); O. Vallee, P. Ranson, and J. Chapelle, J. Phys. B 9, 289 (1976).

2. G. NIENHUIS, Physica 66, 245 (1973).

3. M. Baranger, Phys. Rev. 111, 481 (1958),

4. I. S. GRADSHTEYN and I. M. RYzHIK, Tables of Integrals Series and Products. Academic Press, New York (1965).

5. M. Gell-ManN and M. L. GoldeERGer, Phys. Rev. 91, 398 (1953).

6. F. SCHULler and W. BehMenBuRg, Physics Rep. 12(4), 273 (1974).

7. E. MERZBACHER, Quantum Mechanics. Wiley, New York (1970). 